\title{
Metals at High Redshift
}

\author{
Patrick Petitjean \\ Institut d'Astrophysique de Paris, 98bis Boulevard Arago, 75014 Paris, France \\ email: petitjean@iap.fr
}

\begin{abstract}
The question of how metals are produced in the Universe and where they are located is fundamental for our understanding of galaxy formation and evolution. This question can be best addressed using absorption lines seen in the spectra of remote quasars. It has been realized that the spatial distribution of metals around galaxies and more generally in the Intergalactic Medium is complex, and it is often very difficult to associate one absorption system with one galaxy. Except for possibly in the special case of DLA systems, it may be more appropriate to discuss the clustering properties of different classes of objects and to measure their correlation functions. I illustrate these issues with three examples: the distribution of metals around galaxies at intermediate redshift, the modelling of the clustering of $\mathrm{C}$ IV systems, and the determination of abundances in Damped Lyman- $\alpha$ systems (and in particular the oxygen abundance). Finally, I note that there is a mass-metallicity relation in Damped Lyman- $\alpha$ systems.
\end{abstract}

Keywords. Galaxies: abundances, galaxies: halos, galaxies: evolution, quasars: absorption lines, large-scale structure of universe

\section{Introduction}

It has been rediscovered recently that galaxies could be associated with powerful winds that could expel a non negligible amount of metals into the intergalactic medium. Several lines of argument seem to indicate that this is indeed the case: (i) it has been shown that $\mathrm{C}$ IV and $\mathrm{O}$ VI are present in the intergalactic medium down to $\mathrm{H}$ I optical depths as low as $\tau \sim 1$, corresponding to metallicities (relative to solar) of about $[\mathrm{C} / \mathrm{H}] \sim-3.2$ (Schaye et al. 2003, Aracil et al. 2004); (ii) it seems that, at least in some cases, the intergalactic medium contains less neutral hydrogen than the global average in the vicinity $\left(\sim 0.5 h^{-1} \mathrm{Mpc}\right)$ of Lyman break galaxies at high redshift (Adelberger et al. 2003), which may indicate that supernova-driven winds from these galaxies are able to drive away the intergalactic gas; (iii) star-burst galaxies at low redshift seem to show evidence for winds with velocities of the order of several hundreds of $\mathrm{km} \mathrm{s}^{-1}$ (Heckman et al. 2000).

Although these arguments are suggestive, the overall picture is far from being fully convincing. The exact mechanisms powering these winds are not known and the fate of the gas is unclear (Mac Low \& Ferrara 1999). Although winds may occur more easily in dwarf galaxies, the metals ejected by massive galaxies may dominate the total budget. In any case the spatial distribution of metals around galaxies must be highly complex. It is therefore not a trivial problem to derive this distribution and, in the end, to assess which galaxies are responsible for the enrichment of the IGM and what is the impact of these winds on galaxy formation and evolution.

These issues are discussed using different approaches: (i) the direct detection of objects responsible for the absorption systems gives an indication of the distribution of the gas in the vicinity of galaxies; (ii) the clustering properties of C IV systems give an idea of the large scale distribution of the gas; (iii) determination of metallicities in DLA systems give clues on the star-formation activity in overdense regions. 


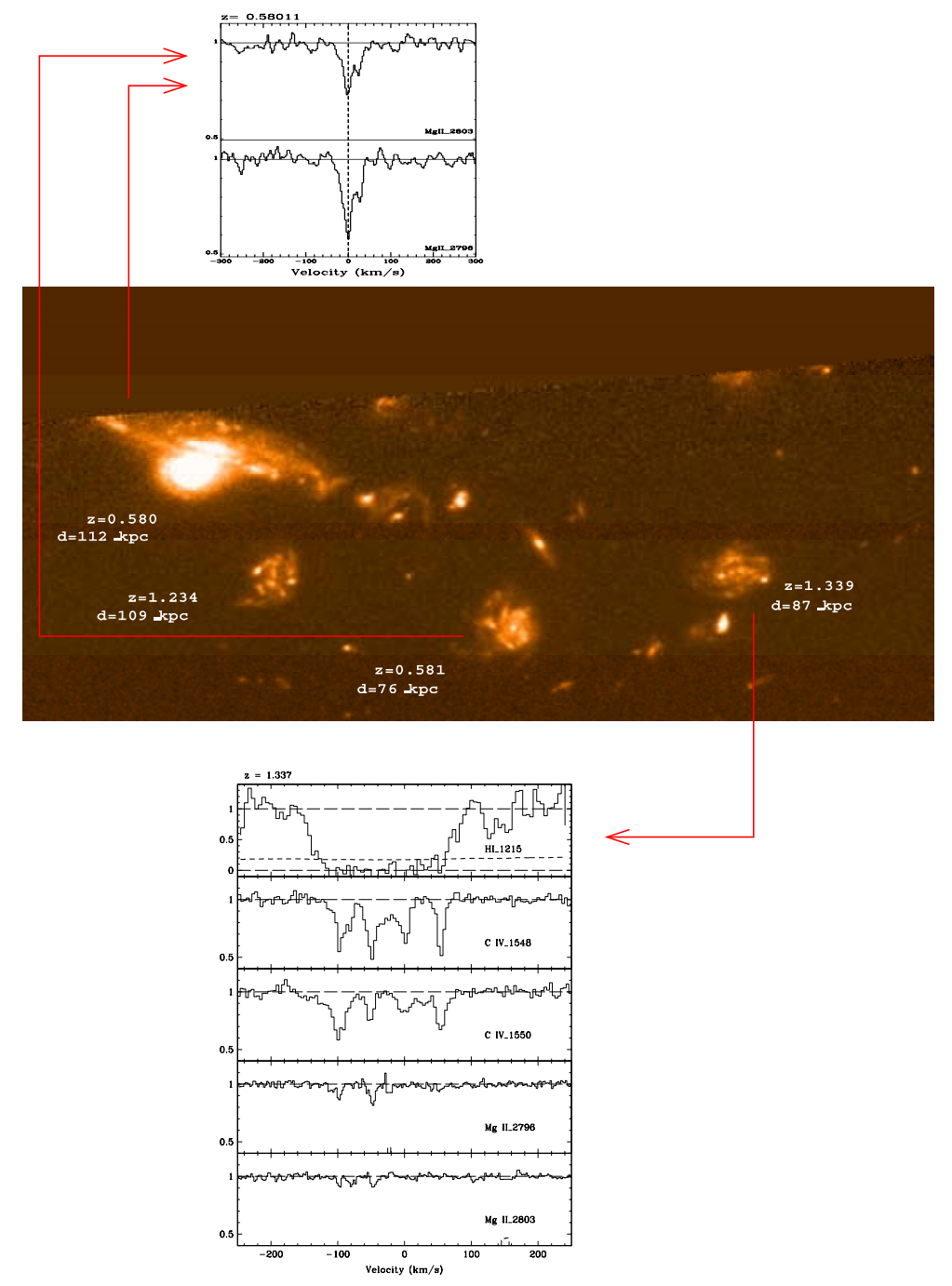

Figure 1. Identification of absorbers in the STIS-HDFS field. The quasar is located about 25 arcsec south of the image. Several weak Mg II absorptions (other species, C IV or H I, are seen as well) are observed at the same redshift as galaxies (here at $z \sim 0.58$ and 1.339). The impact parameters are larger than $100 \mathrm{kpc}$ at $z \sim 1$.

\section{Direct detection of galaxies associated with absorptions}

One way to study the spatial distribution of metals around galaxies is to study the correlation between galaxies and the corresponding absorption line systems detected in the spectrum of background quasars. This is, in addition, the best way to correlate the properties of the galaxy itself (star-formation rate, metallicity, morphology) with those of the gas (ionization state, metallicities and kinematics).

It has been realized that the impact parameters can be very large (e.g. Churchill et al. 2000) implying that at $z \sim 1$, gaseous halos of galaxies have radii larger than $100 \mathrm{kpc}$. If true, it is probable that part of the gas located that far from the central regions is no longer bound to the galaxy. Metals could therefore also trace sheets or filaments from the large scale structure network. To investigate this possibility it is important to go fainter 


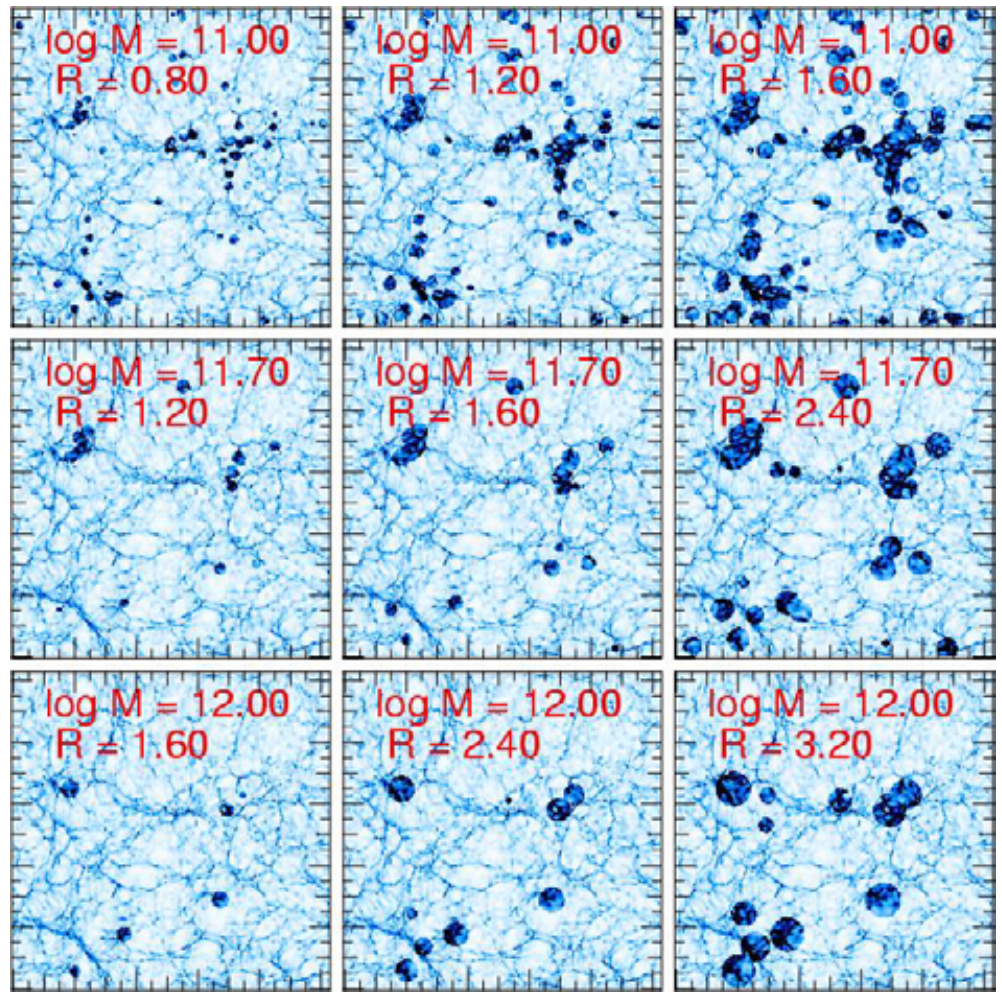

Figure 2. The C IV spatial distribution from a $z=3$ slice in a hydrodynamical simulation meant to reproduce the velocity correlation function and column density distribution of C IV systems. Dark regions are those contained within a distance $R_{\mathrm{s}}$ from a dark matter halo of mass $M_{\mathrm{s}}$, with parameters as labeled in the panels. Prefered models are in the right-side row for which filling factors are $16.1 \%, 16.7 \%$ and $11.6 \%$ from top to bottom respectively (Scannapieco et al. 2005).

than spectroscopic observations can achieve, deriving photometric redshifts for galaxies. This is what we plan to do in several fields of the CFHTLS survey.

\section{Clustering of C IV sytems}

Whereas Mg II is thought to be associated with optically thick gas, C IV arises in gas of higher ionization and is expected to be spread to larger distances from the location of star formation activity. Therefore it is even more difficult to discuss the association of $\mathrm{C}$ IV with galaxies (see an example on Fig. 1). One way to investigate this is to compare the observed clustering properties of C IV absorption lines to the output of $N$ body simulations. We have used $619 \mathrm{C}$ IV absorption components with $N \geqslant 10^{12} \mathrm{~cm}^{-2}$ observed in 19 high signal-to-noise (60-100 per pixel), high resolution $(R=45000)$ quasar spectra. The line-of-sight correlation function $\xi_{\mathrm{CIV}}(v)$ exhibits a steep decline at large separations and a flatter profile below $\sim 150 \mathrm{~km} \mathrm{~s}^{-1}$. These features do not depend on absorber column depths, although there are hints that the overall amplitude of $\xi_{\mathrm{CIV}}$ increases with time over the redshifts range 1.5-3 (see Scannapieco et al. 2005). Carrying out a detailed smoothed particle hydrodynamic simulation $\left(2 \times 320^{3}, 57 \mathrm{Mpc}^{3}\right.$ comoving), we show that the $\mathrm{C}$ IV correlation function can not be reproduced by models in which the IGM metallicity is a constant or a local function of overdensity $\left(Z \propto \Delta^{2 / 3}\right)$. However, 


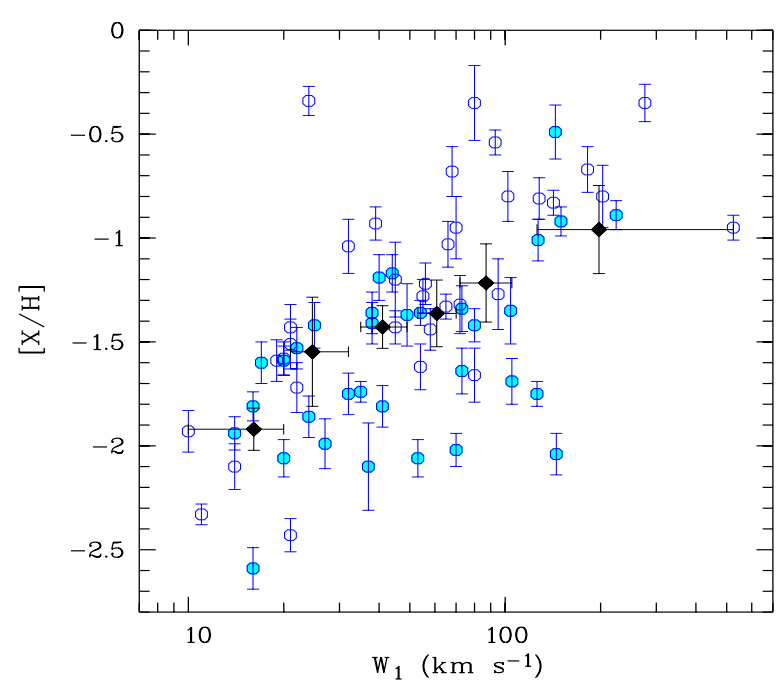

Figure 3. Metallicity relative to solar (either from $\mathrm{Zn}$ or $\mathrm{S}$ or $\mathrm{Si}$ ) in DLAs versus the total width of the absorption lines (as an indication of the corresponding virial velocity). There is a clear correlation revealing a possible relation between the metallicity of the gas and the mass of the halo (Ledoux et al. 2005).

the properties of $\xi_{\mathrm{CIV}}(v)$ are consistent with a model in which metals are confined within bubbles with a typical radius $R_{\mathrm{S}}$ about sources of mass $\geqslant M_{\mathrm{s}}$. We derive best-fit values of $R_{\mathrm{s}} \sim 2$ comoving $\mathrm{Mpc}$ and $M_{\mathrm{s}} \sim 10^{12} \mathrm{M}_{\odot}$ at $z=3$ (see Fig. 2).

\section{Damped Lyman- $\alpha$ Systems}

Damped Lyman- $\alpha$ (DLA) systems are characterized by neutral hydrogen column densities $N(\mathrm{H} \mathrm{I})>10^{20} \mathrm{~cm}^{-2}$. The optical depth is large enough so that hydrogen is neutral. Weak absorption lines from Zn, Fe, S, Si etc. can be measured and accurate metallicities and depletion factors onto dust grains can be derived (metallicities are always smaller than solar with a mean value around $1 / 30$ the solar value).

\subsection{Mass-Metallicity relation}

We have built up a sample of 67 Damped Lyman- $\alpha$ systems with redshift $1.7<z_{\text {abs }}<3.7$ observed with UVES at the VLT. For each system we have measured the average metallicity relative to solar $([\mathrm{X} / \mathrm{H}]$ with $\mathrm{X}=\mathrm{Zn}, \mathrm{S}$, or $\mathrm{Si})$ and the velocity width of low-ionization absorption line profiles $\left(W_{1}\right)$. It has been shown from $N$-body simulations that the later is related to the virial velocity of the dark matter halo in which the DLA system is located : $W_{1} \sim 0.4 v_{\text {vir }}$ (Haehnelt et al. 1998). We find that there is a tight correlation between the metallicity $[\mathrm{X} / \mathrm{H}]$ and the velocity width $W_{1}$ (see Fig. 3 ) detected at more than the $5 \sigma$ significance level (Ledoux et al. 2005). The existence of this correlation over more than two orders of magnitude spread in metallicities, can be understood as a consequence of a mass-metallicity relation for the galaxies responsible for DLA absorption lines. The DLA systems of lowest metallicity would, on average, correspond to the galaxies of lowest mass (e.g., gas rich dwarf galaxies). In turn, these galaxies should have the lowest luminosities. This may explain the result that the few DLAs detected by their Lyman- $\alpha$ emission have higher than average metallicities. 


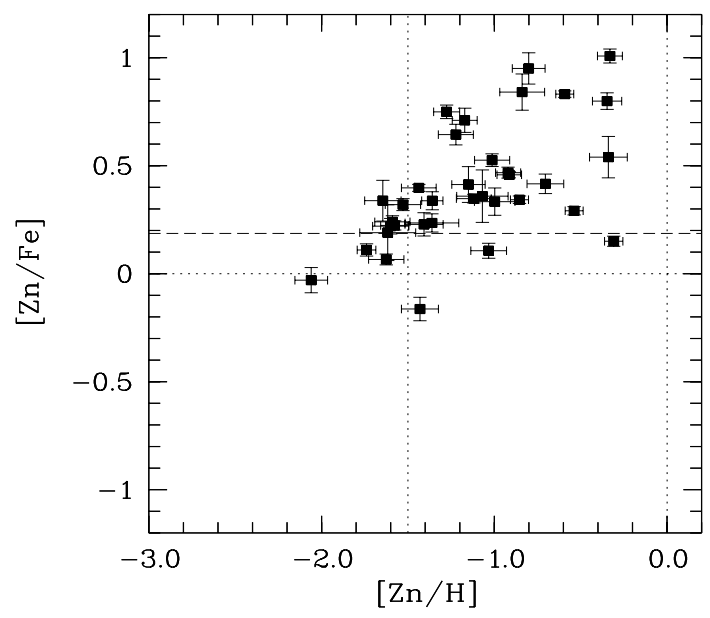

Figure 4. Zinc to Iron relative metallicity versus Zinc metallicity measured in DLAs at high redshift $(z>2)$. All metallicities are considered relative the the solar values. The correlation observed for $[\mathrm{Zn} / \mathrm{H}]>-1.5$ is a clear indication of the presence of dust, Iron being more depleted at higher metallicity.

\subsection{Abundance measurements}

Metallicities and dust content have been derived from Zinc and Iron measurements as in our galaxy, Zinc is observed to trace the gaseous abundances whereas Iron is observed to deplete heavily onto dust grains. The abundance ratio $[\mathrm{Zn} / \mathrm{Fe}]$ is considered as an indicator of the presence of dust. It can be seen on Fig. 4 that there is a correlation for $[\mathrm{Zn} / \mathrm{H}]>-1.5([\mathrm{Zn} / \mathrm{H}]$ is measured relative to the solar value) between $[\mathrm{Zn} / \mathrm{Fe}]$ and $[\mathrm{Zn} / \mathrm{H}]$. This is a clear indication of the presence of dust, Iron being more depleted at higher metallicity.

Some abundance measurements are very important for the discussion of the star formation activity at high redshift. In particular Oxygen is a key-element of nucleosynthesis. Measurements are difficult because most of the time only the strong O I $\lambda 1302$ line is observed. However, reliable measurements can be done at low metallicities (Fig. 5). It can be seen that there is a plateau at $[\mathrm{O} / \mathrm{Fe}] \sim+0.35$ for $-3<[\mathrm{O} / \mathrm{H}]<-1.5$. Another difficult but important element is phosphorus which seems to have a much eratic behavior (see Fig. 6).

\section{Acknowledgements}

I would like to thank our collaborators for allowing me to refer to common works: B. Aracil, R. Carswell, F. Coppolani, M. Haehnelt, C. Ledoux, M. Longhetti, C. Pichon, E. Rollinde, E. Scannapieco, R. Srianand, F. Stoehr. Data presented here have been obtained at the ESO-VLT observatory on Mount Paranal, Chile.

\section{References}

Adelberger, K.L., et al., 2003, ApJ, 584, 45

Aracil, B., et al., 2004, A\&A, 419, 811

Churchill, C., et al., 2000, ApJ, 543, 577

Haehnelt, M., Steinmetz, M., \& Rauch, M. 1998, ApJ, 495, 647

Heckman, T.M., et al., 2000, ApJS, 129, 493

Ledoux, C., Petitjean, P, Møller, P., Fynbo, J., \& Srianand, R. 2005, astrop-ph/0504402 


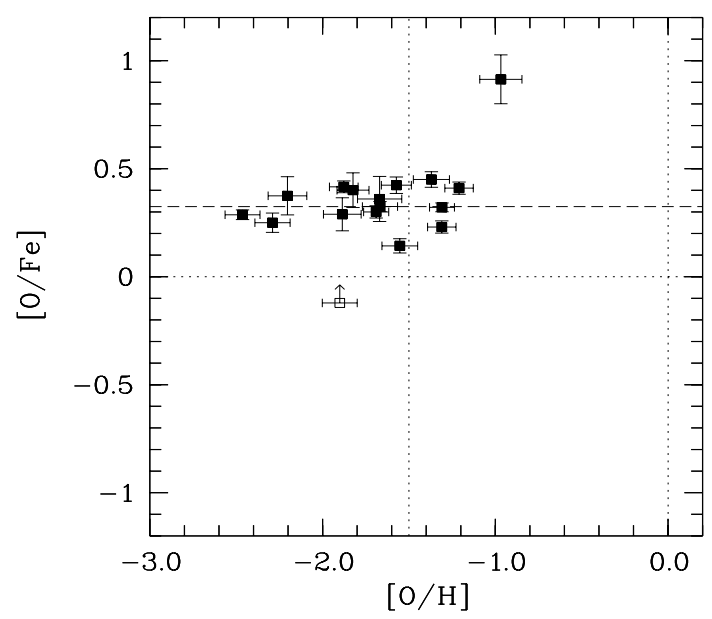

Figure 5. Oxygen to Iron relative metallicity versus Oxygen metallicity (relative to solar) measured in DLAs at high redshift $(z>2)$.

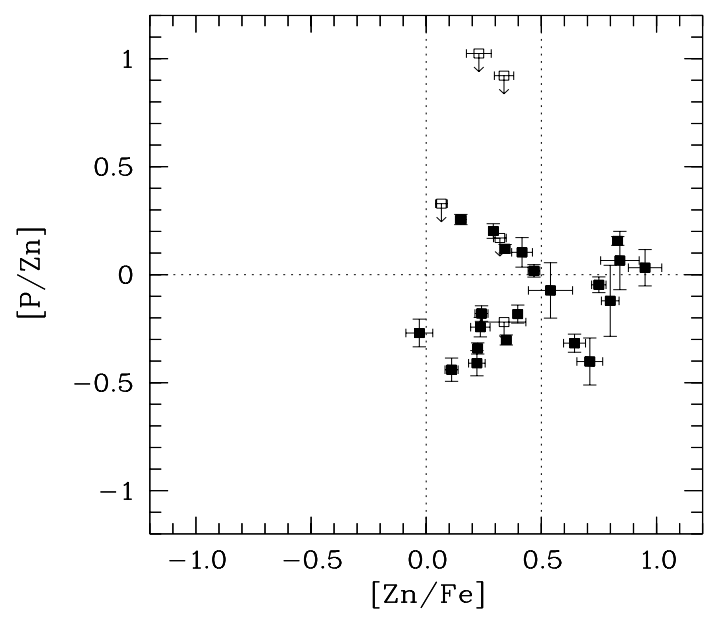

Figure 6. Phosphorus to Zinc relative metallicity versus Zinc to Iron relative metallicity as measured in DLAs at high redshift $(z>2)$. The later abundance ratio is an indicator of the depletion into dust grains as in the ISM of our Galaxy, Iron is observed to be highly depleted from the gas phase when Zinc is not.

Mac Low, M.M. \& Ferrara, A. 1999, ApJ, 513, 142

Scannapieco, E., Pichon, C., Aracil., B., et al. 2005, astro-ph/0503001

Schaye, J., et al., 2003, ApJ, 596, 768 